\title{
Nanoparticles in fossil and mineral fuel sectors and their impact on environment and human health: A review and perspective
}

Luis F.O., M. Santosh, Michael Schindler, Juciano Gasparotto, Guilherme L. Dotto, Marcos L.S. Oliveira, Michael F. Hochella jr.

https://doi.org/10.1016/i.gr.2020.12.026

Palabras clave

Nanoparticles and nanominerals, Environmental impacts, Environmental contaminants, Mining activities, Human health

Abstract

Nanoscience and technology have enabled better insights into the environmental and health impacts arising from the mining, production and use of fossil and mineral fuels. Here we provide an overview of the nanoscience-based applications and discoveries concerning coal and mineral fuel (i.e., uranium-containing minerals) mining, refining/production, use, and disposal of wastes. These processes result in massive nanoparticle release and secondary nanoparticle generation which have highly significant environmental implications and human health consequences on local, regional, and even global levels. Until recently, very little was known about nanoparticle fractions. Recent advancements and sophistications enable us to detect, collect and study these materials which are roughly $1 \mathrm{~nm}(0.001 \mu \mathrm{m})$ up to several tens of nanometers in size. These materials are known to behave differently (chemically, electrically, and mechanically), relative to their macroscopic equivalents. This is what makes nanoscience fascinating and difficult to predict, underscoring the importance of this emerging new field. For example, nanoparticles associated with coal and mineral fuel influence the release, uptake, and transportation of hazardous elements associated with mining, processing, and waste storage in the surrounding areas. This includes long distance transport down streams, rivers, and eventually to oceans such as from coal and uranium mine drainages. In terms of human health, in all phases of mining, production/refining, use, and waste disposal, the associated nanoparticles can be acquired through oral ingestion, inhalation, and dermal absorption. Inhalation has been shown to be particularly damaging, where lung, heart, kidney, and brain diseases are prevalent.

Relative to all other fields of science and engineering associated with coal and mineral fuel mining, production, use, and clean-up efforts, nanoscience, although a much newer field then the rest by comparison, is still greatly under-represented and under-utilized. There is also a continuing gap 
between what we so far know about the behavior of nanoparticles, and what remains to be discovered. 